\title{
On martingale measures and pricing for continuous bond-stock market with stochastic bond
}

\author{
Nikolai Dokuchaev \\ Department of Mathematics \& Statistics, Curtin University, \\ GPO Box U1987, Perth, 6845 Western Australia \\ Submitted: August 3, 2011. Revised: September 30, 2014
}

\begin{abstract}
This papers addresses the stock option pricing problem in a continuous time market model where there are two stochastic tradable assets, and one of them is selected as a numéraire. It is shown that the presence of arbitrarily small stochastic deviations in the evolution of the numéraire process causes significant changes in the market properties. In particular, an equivalent martingale measure is not unique for this market, and there are non-replicable claims. The martingale prices and the hedging error can vary significantly and take extreme values, for some extreme choices of the equivalent martingale measures. Some rational choices of the equivalent martingale measures are suggested and discussed, including implied measures calculated from observed bond prices. This allows to calculate the implied market price of risk process.
\end{abstract}

Key words: martingale pricing, random numéraire, stochastic bond price, incomplete market, hedging error.

JEL classification: G13

MSC 2010 classification: 91G20

\section{Introduction}

This paper addresses the stock option pricing problem in a continuous time market model.

We consider the case where there are two stochastic tradable assets, and one of them is 
selected as a numéraire. This setting corresponds to a generalized Black-Scholes model where stochastic deviations in the bond prices are allowed. The classical Black and Scholes model includes a bond or money market account with the price $B(t)$ and a single risky asset with the price $S(t)$; the process $B(t)$ is assumed to be non-random or risk-free and is used as a numéraire, and $S(t)$ is assumed to be a stochastic Itô process with constant volatility. This is a so-called complete market where any claim can be replicated and where there is a unique equivalent martingale (risk-neutral) measure equivalent to the historical measure; the price of a derivative is defined via the expectation of the discounted payoff by this unique equivalent martingale measure.

The pricing of derivatives is usually more difficult for so-called incomplete market models where an equivalent martingale measure is not unique; see, e.g., El Karoui and Quenez (1995).

Some important examples of market incompleteness arise when the money market account is assumed to have a martingale part. Cheng (1991), Kim and Kunitomo (1999), Benninga et al. (2002), and Back (2010), considered an incomplete modification of the Black-Scholes model where $B(t)$ was an Itô process. The cited papers considered martingale pricing method where the option price is calculated as the expectation of the discounted claim under some equivalent risk-neutral measure (martingale measure) such that the discounted stock price $S(t) / B(t)$ is a martingale on a given time interval $[0, T]$ under this measure. Cheng (1991) analyzed only one equivalent martingale measure among all equivalent martingale measures; see Example 2.1 (iv). Benninga et al. (2002) considered a multi-stock market under requirements that make the choice of an equivalent martingale measure unique in the case of a single stock and stochastic bond; see Example 2.1 (iii). Geman et al. (1995) considered pricing of replicable claims only. Kim and Kunitomo (1999) studied asymptotic properties of this price with respect to a particular equivalent martingale measure. In the cited works, the pricing was studied for certain selected measures, and the impact of non-uniqueness of an equivalent martingale measure was not discussed, as well as the presence of non-replicable claims.

For portfolio selection problems, related questions arise in the setting with a random numéraire; see, e.g., Karatzas and Kardaras (2007), Kardaras (2010), Becherer (2010).

In this paper, we revisited the pricing problem for options for a market with two tradable stochastic assets. We consider a model with a continuum of different equivalent martingale 
measures and with claims that cannot be replicated, even when the appreciation rate and volatility coefficients are constant (Proposition [3.2). We investigate the impact of the presence of different equivalent martingale measures and non-replicable claims. Our model is close to the model from Cheng (1991) with a modification that ensures the existence of many equivalent martingale measures. Cheng (1991) studied an impact of the absence of an equivalent martingale measure in the setting with a stochastic bond price $B(t)$ such that $B(T)=1$. In this case, the appreciation rate of the discounted stock price is imploding when terminal time is approached, and the Novikov's condition of existence of an equivalent martingale measure is not satisfied. Our setting removes this feature; we consider a stochastic numéraire without restrictions on the terminal price. This could be close to the model from Cheng (1991) if one considers a stochastic bond with the price $B(t)$ maturing at $T+\varepsilon$, i.e., such that $B(T+\varepsilon)=1$, for an arbitrarily small $\varepsilon>0$. We suggest some criterions of replicability (Theorems 3.1 and 3.2). We investigated pricing of zero coupon bonds for this market, and derived an equation for the associated risk-free rate (equations (4.1)-(4.5) in Section 4 and 7). Using these results, we obtained that, for a market model with stochastic numéraire, it is possible to estimate the market price of risk and therefore the appreciation rate of the stock price process as an implied parameter inferred from a single observed market price of a zero coupon bond. This is a novell result, since the existing literature does not consider implied appreciation rate; see discussion and citations in Section 4.

In addition, we found that there are interesting features that makes this model different from the models with non-random numéraire and with the incompleteness caused by the randomness of the volatility. Apparently, arbitrarily small deviations in the numéraire coefficients cause significant changes in the market properties. With these deviations, the martingale prices and the second moments of the hedging error vary significantly and take extreme values for some extreme choices of equivalent martingale measures that represent "extreme" beliefs of market agents (Theorems [5.1/5.4). This can be interpreted as the following: among the variety of equivalent martingale measures, there are some measures that represent extreme beliefs and can be excluded from the analysis. We suggest a parametrisation of the set of equivalent martingale measures that helps to select reasonable equivalent martingale measures, including a measure implied form observed prices of zero coupon bonds 
(Theorems 6.1 ). We discuss some particular choices of these measures, including a measure implied form observed prices of zero coupon bonds and a measure that ensures local risk minimizing hedging strategy (Examples 6.16.4 and Theorem 6.2).

The rest of the paper is organized as follows. In Section 2, we describe the model setting. In Section 3, we discuss replicability of claims and the hedging errors. In Section 5, we show that the prices can take extreme values for some choices of martingale measures. In Section 4. we suggest a method of calculation the implied market price of risk for a case of complete market and review some related literature. In Section 6, we discuss selection of the equivalent martingale measures. In Section 7, we discuss calculation of the implied market price of risk for a more general case of an incomplete market. In Section 8, parabolic equations are derived for the price, for the hedging strategy, and for the hedging error, in a Markovian setting. The proofs are in the Appendix.

\section{Model setting}

We consider the diffusion model of a securities market consisting of two tradable assets with the prices $S(t)$ and $B(t), t \geq 0$. The prices evolve as

$$
d S(t)=S(t)(a(t) d t+\sigma(t) d w(t)+\widehat{\sigma}(t) d \widehat{w}(t)), \quad t>0,
$$

and

$$
d B(t)=B(t)(\alpha(t) d t+\rho(t) d w(t)+\widehat{\rho}(t) d \widehat{w}(t))
$$

We assume that $W(t)=(w(t), \widehat{w}(t))$ is a standard Wiener process with independent components on a given standard probability space $(\Omega, \mathcal{F}, \mathbf{P})$, where $\Omega$ is a set of elementary events, $\mathbf{P}$ is a probability measure, and $\mathcal{F}$ is a $\mathbf{P}$-complete $\sigma$-algebra of events. The initial prices $S(0)>0$ and $B(0)>0$ are given constants.

We consider this model as an extension of the classical bond and stock market model, where a bond with the price $B(t)$ is used as a numéraire, and its price evolves as

$$
\frac{d B}{d t}(t)=\alpha(t) B(t)
$$

Equation (2.1) for stochastic numéraire is a generalization of (2.2); one may say that equation (2.1) represents a modification of equation for a risk-free asset that takes into account 
possibility of stochastic disturbances in the return rate. In this setting, $B(t)$ is not exactly a risk-free asset. However, if the processes $\rho(t)$ and $\widehat{\rho}(t)$ are small in some norm, then (2.1) can be considered as the equation for the money market account with small deviations (see an example in Section 3 below). In particular, the conditions on the coefficients imposed below allow the case where $\rho(t) \equiv \widehat{\rho}(t)=\varepsilon$ for an arbitrarily small $\varepsilon>0$. We show below that the presence of arbitrarily small deviations in (2.1) changes dramatically the properties of the market model (see Section 5 ).

If $\operatorname{ess}_{\sup _{t, \omega}}(|\widehat{\sigma}(t, \omega)|+|\widehat{\rho}(t, \omega)|)>0$, we denote by $\mathbb{F}=\left(\mathcal{F}_{t}\right)_{t \geq 0}$ the filtration generated by the process $W=(w, \widehat{w})$. If $\operatorname{ess}_{\sup }(|\widehat{\sigma}(t, \omega)|+|\widehat{\rho}(t, \omega)|)=0$, we denote by $\mathbb{F}=\left(\mathcal{F}_{t}\right)_{t \geq 0}$ the filtration generated by the process $w$ only. In both cases, $\mathcal{F}_{0}$ is trivial, i.e., it is the $\mathbf{P}$-augmentation of the set $\{\emptyset, \Omega\}$.

We assume that the process $\mu(t)=(a(t), \sigma(t), \widehat{\sigma}(t), \alpha(t), \rho(t), \widehat{\rho}(t))$ is $\mathbb{F}$-adapted and bounded.

Let $\widetilde{\sigma}=\sigma-\rho$ and $\widetilde{\rho}=\widehat{\sigma}-\widehat{\rho}$. We assume that there exists $c>0$ such that either $|\widetilde{\sigma}(t, \omega)| \geq c$ a.e. or $|\widetilde{\rho}(t, \omega)|>c$ a.e.

\section{Discounted stock price and equivalent martingale measures}

Let $\widetilde{S}(t) \triangleq S(t) / B(t)$. By Itô formula, it follows that this process evolves as

$$
\begin{aligned}
& d \widetilde{S}(t)=\widetilde{S}(t)(\widetilde{a}(t) d t+\widetilde{\sigma}(t) d w(t)+\widetilde{\rho}(t) d \widehat{w}(t)), \\
& \widetilde{S}(0)=S(0) / B(0),
\end{aligned}
$$

where

$$
\widetilde{a} \triangleq a-\alpha+\rho^{2}+\widehat{\rho}^{2}-\sigma \rho-\widehat{\sigma} \widehat{\rho} .
$$

Let $V(t)=\left(V_{1}(t), V_{2}(t)\right)^{\top}=(\widetilde{\sigma}(t), \widetilde{\rho}(t))^{\top}$ and $\widehat{V}(t)=\left(\widehat{V}_{1}(t), \widehat{V}_{2}(t)\right)^{\top}=(\rho(t), \widehat{\rho}(t))^{\top}$. These processes take values in $\mathbf{R}^{2}$. By the assumptions, ess $\inf _{t, \omega}|V(t, \omega)|>0$.

Definition 2.1 Let $\mathcal{T}$ be the set of bounded $\mathbb{F}$-adapted processes $\theta(t)=\left(\theta_{1}(t), \theta_{2}(t)\right)^{\top}$ with values in $\mathbf{R}^{2}$ such that $\theta_{1}(t) \widetilde{\sigma}(t)+\theta_{2}(t) \widetilde{\rho}(t)=\widetilde{a}(t)$, i.e., $V(t)^{\top} \theta(t)=\widetilde{a}(t)$.

For $\theta \in \mathcal{T}$, set

$$
\mathcal{Z}_{\theta}=\exp \left(-\int_{0}^{T} \theta(s)^{\top} d W(s)-\frac{1}{2} \int_{0}^{T}|\theta(s)|^{2} d s\right) .
$$


Our standing assumptions imply that $\mathbf{E} \mathcal{Z}_{\theta}=1$. Define the probability measure $\mathbf{P}_{\theta}$ by $d \mathbf{P}_{\theta} / d \mathbf{P}=\mathcal{Z}_{\theta}$; this measure is equivalent to the measure $\mathbf{P}$. Let $\mathbf{E}_{\theta}$ be the corresponding expectation.

Let

$$
W_{\theta}(t)=\left(\begin{array}{c}
W_{\theta 1}(t) \\
W_{\theta 2}(t)
\end{array}\right)=\int_{0}^{t} \theta(s) d s+W(t) .
$$

By Girsanov's Theorem, $W_{\theta}$ is a standard Wiener process in $\mathbf{R}^{2}$ under $\mathbf{P}_{\theta}$.

For $\theta \in \mathcal{T}$, equation (2.4) can be rewritten as

$$
d \widetilde{S}(t)=\widetilde{S}(t) V(t)^{\top} d W_{\theta}(t)
$$

Remark 2.1 Clearly, the set $\mathcal{T}$ has more than one element; it is a linear manifold. Therefore, the selection of the process $\theta(t)$ and the measure $\mathbf{P}_{\theta}$, is not unique.

Example 2.1 (i) If $\widetilde{\rho} \equiv 0$, then the process $\theta_{1}(t)$ is uniquely defined as $\theta_{1}(t)=\widetilde{\sigma}(t)^{-1} \widetilde{a}(t)$. If, in addition, the process $\widetilde{\sigma}(t)$ is non-random, then the process $\widetilde{S}(t)$ has the same distribution under $\mathbf{P}_{\theta}$ for all $\theta \in \mathcal{T}$.

(ii) If $\widetilde{\sigma} \equiv 0$, then the process $\theta_{2}(t)$ is uniquely defined as $\theta_{2}(t)=\widetilde{\rho}(t)^{-1} \widetilde{a}(t)$.

(iii) Benninga et al. (2002) considered a multi-stock market with special requirements for the equivalent martingale measure. For our special case of a single stock and a stochastic numéraire, these requirements leads to a unique equivalent martingale measure such that the process $\left(\widetilde{S}(t), \exp \left(\int_{0}^{t} k(s) d s\right) B(t)^{-1}\right)$ is a martingale, for a given process $k(t) \geq 0$.

(iv) Let $z(t)=\int_{0}^{t}|V(s)|^{-1} V(s)^{\top} d W(s)$; by Lévy's characterization theorem, it is an onedimensional Wiener process. Let $q(t)=|V(s)|^{-1} \widetilde{a}(t)$. By the assumptions, it is a bounded process. Let $\widehat{z}(t)=\int_{0}^{t} q(s) d s+z(t)$. We have that $V(t)^{\top} d W(t)=|V(t)| d z(t)$ and

$$
d \widetilde{S}(t)=\widetilde{S}(t)(\widetilde{a}(t) d t+|V(t)| d z(t))=\widetilde{S}(t)|V(t)| d \widehat{z}(t)
$$


By Girsanov's Theorem, there is an equivalent martingale measure $\widehat{\mathbf{P}}$ such that $\widehat{z}(t)$ a Wiener process under $\widehat{\mathbf{P}}$. It follows that the process $\widetilde{S}(t)$ is an equivalent martingale under $\widehat{\mathbf{P}}$. This martingale measure was studied in Cheng (1991).

Let $\mathcal{Y}_{\theta}$ be the set of all $\mathbb{F}$-adapted measurable processes with values in $\mathbf{R}^{2}$ that are square integrable on $[0, T] \times \Omega$ with respect to $\ell_{1} \times \mathbf{P}_{\theta}$, where $\ell_{1}$ is the Lebesgue measure.

Let $\mathcal{H}_{\theta}$ be the Hilbert space formed as the completion of the set of $\mathbb{F}$-adapted measurable processes $y(t)$ such that $\|y\|_{\mathcal{H}_{\theta}}=\left(\mathbf{E}_{\theta} \int_{0}^{T}|\widetilde{S}(t) y(t)|^{2} d t\right)^{1 / 2}<+\infty$.

\section{Wealth and discounted wealth}

Let $X(0)>0$ be the initial wealth at time $t=0$ and let $X(t)$ be the wealth at time $t>0$.

We assume that the wealth $X(t)$ at time $t \geq 0$ is

$$
X(t)=\beta(t) B(t)+\gamma(t) S(t)
$$

Here $\beta(t)$ is the quantity of the numéraire portfolio, $\gamma(t)$ is the quantity of the stock portfolio, $t \geq 0$. The pair $(\beta(\cdot), \gamma(\cdot))$ describes the state of the securities portfolio at time $t$. Each of these pairs is called a strategy.

Definition 2.2 Let $\theta \in \mathcal{T}$ be given. A pair $(\beta(\cdot), \gamma(\cdot))$ is said to be an admissible strategy under $\mathbf{P}_{\theta}$ if the processes $\beta(t)$ and $\gamma(t)$ are progressively measurable with respect to the filtration $\left(\mathcal{F}_{t}\right)_{t \geq 0}$ and such that

$$
\mathbf{E}_{\theta} \int_{0}^{T} \widetilde{S}(t)^{2} \gamma(t)^{2} d t<+\infty
$$

Definition 2.3 Let $\theta \in \mathcal{T}$ be given. A pair $(\beta(\cdot), \gamma(\cdot))$ that is an admissible strategy under $\mathbf{P}_{\theta}$ is said to be a self-financing strategy, if there exists a sequence of Markov times $\left\{T_{k}\right\}_{k=1}^{\infty}$ with respect to $\left(\mathcal{F}_{t}\right)_{t \geq 0}$ such that $0 \leq T_{k} \leq T_{k+1} \leq T$ for all $k, T_{k} \rightarrow T$ as $k \rightarrow+\infty$ a.s., and

$$
\mathbf{E}_{\theta} \int_{0}^{T_{k}}\left(\beta(t)^{2} B(t)^{2}+S(t)^{2} \gamma(t)^{2}\right) d t<+\infty, \quad k=1,2, \ldots
$$

and the corresponding wealth $X(t)=\gamma(t) S(t)+\beta(t) B(t)$ is such that

$$
d X(t)=\gamma(t) d S(t)+\beta(t) d B(t) .
$$


Note that condition (2.9) ensures that the stochastic differentials in (2.10) are well defined.

Let $\widetilde{X}(t) \triangleq X(t) / B(t)$. The process $\widetilde{X}(t)$ is said to be the discounted wealth.

The following lemma is known; see, e.g., Geman et al. (1995), Jamshidian (2008), Bielecki et al. (2009).

Lemma 2.1 If a strategy $(\beta(t), \gamma(t))$ is self-financing and admissible under $\mathbf{P}_{\theta}$ for some $\theta \in \mathcal{T}$, then, for the corresponding discounted wealth,

$$
d \widetilde{X}(t)=\gamma(t) d \widetilde{S}(t)
$$

Remark 2.2 Since we assume that the coefficients for the equations for $S(t)$ and $B(t)$ are bounded, it follows from Lemma 2.1 that if (2.8) holds for some $\theta$ then $\mathbf{E}_{\theta} \widetilde{X}(T)^{2}<+\infty$ for this $\theta$.

Lemma 2.2 For every $\theta \in \mathcal{T}$, the processes $\widetilde{X}(t)$ and $\widetilde{S}(t)$ are martingales under $\mathbf{P}_{\theta}$ with respect to $\left(\mathcal{F}_{t}\right)_{t \geq 0}$, i.e., $\mathbf{E}_{\theta}\left\{\widetilde{S}(T) \mid \mathcal{F}_{t}\right\}=\widetilde{S}(t)$ and $\mathbf{E}_{\theta}\left\{\tilde{X}(T) \mid \mathcal{F}_{t}\right\}=\widetilde{X}(t)$.

Remark 2.3 Consider an European option with the payoff $B(T) \xi$, where $\xi$ is an $\mathcal{F}_{T}$-measurable random variable. For any $\theta \in \mathcal{T}$ such that $\mathbf{E}_{\theta} \xi^{2}<+\infty$, the option price $\mathbf{E}_{\theta} \xi$ is an arbitragefree price.

\section{On the replicability of contingent claims}

For $\theta \in \mathcal{T}$, let $\mathcal{X}_{\theta}$ be the subspace of $L_{2}\left(\Omega, \mathcal{F}_{T}, \mathbf{P}_{\theta}\right)$ consisting of all $\zeta \in L_{2}\left(\Omega, \mathcal{F}_{T}, \mathbf{P}_{\theta}\right)$ such that there exists an admissible self-financing strategy $(\beta(\cdot), \gamma(\cdot))$ under $\mathbf{P}_{\theta}$ and the corresponding wealth process $X(t)$ such that $X(0)=0$ and $X(T)=B(T) \zeta$.

Let $\mathcal{X}_{\theta}^{\perp} \triangleq\left\{\eta \in L_{2}\left(\Omega, \mathcal{F}_{T}, \mathbf{P}_{\theta}\right): \quad \mathbf{E}_{\theta} \eta=0, \quad \mathbf{E}_{\theta}[\zeta \eta]=0\right.$ for all $\left.\zeta \in \mathcal{X}_{\theta}\right\}$.

Let $\xi \in L_{2}\left(\Omega, \mathcal{F}_{T}, \mathbf{P}_{\theta}\right)$. By the Martingale Representation Theorem, we have that, for some uniquely defined $U_{\theta} \in \mathcal{Y}_{\theta}$ and $c_{\theta} \in \mathbf{R}$,

$$
\xi=c_{\theta}+\int_{0}^{T} U_{\theta}(t)^{\top} d W_{\theta}(t) .
$$

In addition, it follows from the properties of closed subspaces in Hilbert spaces that $\xi$ can be represented via Föllmer-Schweizer decomposition

$$
\xi=c_{\theta}+I_{\theta}+R_{\theta}
$$


Here $c_{\theta}=\mathbf{E}_{\theta} \xi, R_{\theta} \in \mathcal{X}_{\theta}^{\perp}$, and

$$
I_{\theta}=\int_{0}^{T} \gamma_{\theta}(t) d \widetilde{S}(t) \in \mathcal{X}_{\theta}
$$

for some $\gamma_{\theta} \in \mathcal{H}_{\theta}$, i.e., it is the terminal discounted wealth $\tilde{X}(T)$ for some admissible selffinancing strategy $\left(\beta_{\theta}(\cdot), \gamma_{\theta}(\cdot)\right)$ under $\mathbf{P}_{\theta}$ and for the initial wealth $X(0)=0$. Therefore, a contingent claim $B(T) \xi$ can be decomposed as $B(T)\left(\widetilde{\xi}_{\theta}+R_{\theta}\right)$, where $B(T) R_{\theta}$ is the hedging error and where $B(T) \widetilde{\xi}_{\theta}$ is a replicable part such that $\widetilde{\xi}_{\theta}=c_{\theta}+\int_{0}^{T} \gamma_{\theta}(t) d \widetilde{S}(t)$.

Let us express $\gamma_{\theta}$ via $U_{\theta}$.

Proposition 3.1 Let $\xi \in L_{2}\left(\Omega, \mathcal{F}_{T}, \mathbf{P}_{\theta}\right)$, and let $U_{\theta}$ be defined by (3.1). Let

$$
\nu_{\theta}(t)=U_{\theta}(t)^{\top} V(t) /|V(t)|^{2}, \quad \eta_{\theta}(t)=U_{\theta}(t)-\nu_{\theta}(t) V(t)
$$

Then

$$
\eta_{\theta}(t)^{\top} V(t) \equiv 0
$$

and (3.2) holds with

$$
I_{\theta}=\int_{0}^{T} \nu_{\theta}(t) V(t)^{\top} d W_{\theta}(t), \quad R_{\theta}=\int_{0}^{T} \eta_{\theta}(t)^{\top} d W_{\theta}(t) .
$$

Further, (3.3) holds with

$$
\gamma_{\theta}(t)=\nu_{\theta}(t) \widetilde{S}(t)^{-1}
$$

Proof. It suffices to observe that $\eta_{\theta} \in \mathcal{Y}_{\theta}$, and that $\nu_{\theta} V$ is the projection of $U_{\theta}$ on $V$. It follows that $R_{\theta} \in \mathcal{X}_{\theta}^{\perp}$. The uniqueness follows from the properties of orthogonal subspaces of a Hilbert space.

The following statement follows from the non-uniqueness of the equivalent martingale measures and The 2nd Fundamental Theorem of Asset Pricing.

Proposition 3.2 Assume $\widetilde{\rho}(\cdot) \neq 0$, i.e., it is not an identically zero process. Then the set $\mathcal{X}_{\theta}^{\perp}$ contains non-zero elements, i.e., $\sup _{\eta \in \mathcal{X}_{\theta}^{\perp}} \mathbf{E}_{\theta}|\eta|>0$.

By this proposition, the hedging error $R_{\theta}$ is non-zero in the general case. In other word, a contingent claim of a general type is not replicable. For completeness, we will give in the Appendix the proof adjusted to our model. 
Let us describe some cases of replicability.

Let $\left(\mathcal{F}_{t}^{w}\right)_{t \geq 0}$ be the filtration generated by the process $w(t)$, and let $\left(\mathcal{F}_{t}^{\widetilde{S}}\right)_{t \geq 0}$ be the filtration generated by the process $\widetilde{S}(t)$.

Theorem 3.1 Assume that the processes $\widetilde{\sigma}(t)$ and $\widetilde{\rho}(t)$ are non-random. Then the claims $B(T) \xi$ are replicable for $\xi \in L_{2}\left(\Omega, \mathcal{F}_{T}^{\widetilde{S}}, \mathbf{P}_{\theta}\right)$ for any $\theta \in \mathcal{T}$. More precisely, there exists an $\mathbb{F}$-adapted process $\gamma(t)$ such that $\mathbf{E}_{\theta} \int_{0}^{T} \gamma(t)^{2} \widetilde{S}(t)^{2} d t<+\infty$ and $\xi=\mathbf{E}_{\theta} \xi+\int_{0}^{T} \gamma(t) d \widetilde{S}(t)$.

\section{The case of complete market}

Note that the market described in Theorem 3.1 is incomplete since there are claims that cannot be replicated. The following theorem describes an important special case when the market is complete.

Theorem 3.2 Assume that the processes $\widetilde{a}(t)$ and $\widetilde{\sigma}(t)$ are adapted to the filtration $\left(\mathcal{F}_{t}^{w}\right)_{t \geq 0}$ generated by the process $w(t)$, and that $\widetilde{\rho}(t) \equiv 0$, i.e., it is an identically zero process. Then $\theta_{1}(t)=\widetilde{a}(t) \widetilde{\sigma}(t)^{-1}$ for any $\theta \in \mathcal{T}$, and the claims $B(T) \xi$ are replicable for $\xi \in L_{2}\left(\Omega, \mathcal{F}_{T}^{w}, \mathbf{P}_{\theta}\right)$.

\section{The implied market price of risk: the case of complete mar- ket}

Up to the end of this section, we assume that that $\widehat{\sigma}(t) \equiv \widehat{\rho}(t) \equiv 0$. In this case, by the definition of $\mathcal{F}_{t}$, we have that $\mathcal{F}_{t}=\mathcal{F}_{t}^{w}$, and the assumptions of Theorem 3.2 are satisfied.

Lemma 4.1 The claim $\xi \equiv \$ 1$ is replicable in the following sense: for any $t \in[0, T)$, there exists an $\mathbb{F}$-adapted process $\gamma(t)$ such that $\mathbf{E}_{\theta} \int_{0}^{T} \gamma(t)^{2} \widetilde{S}(t)^{2} d t<+\infty$ and

$$
B(T)^{-1}=\mathbf{E}_{\theta} B(T)^{-1}+\int_{0}^{T} \gamma(t) d \widetilde{S}(t) .
$$

It can be noted that, by the definitions, under the assumption of Lemma 4.1, the processes $a(t), \sigma(t), \alpha(t)$, and $\rho(t)$, are $\mathcal{F}_{t}^{w}$-adapted. In addition, $\theta_{1}(t)=\widetilde{a}(t) \widetilde{\sigma}(t)^{-1}$ for any $\theta \in \mathcal{T}$.

Under the assumptions of Lemma 4.1, the value $\mathbf{E}_{\theta} B(T)^{-1}$ represents the price at time $t=0$ of a zero-coupon bond with the payoff $\$ 1$ at the maturity time $T$. The value $\tilde{X}(t)=$ $\mathbf{E}_{\theta}\left\{B(T)^{-1} \mid \mathcal{F}_{t}\right\}$ represents the discounted wealth for the replicating strategy, and the value

$$
P(t, T)=B(t) \tilde{X}(t)=B(t) \mathbf{E}_{\theta}\left\{B(T)^{-1} \mid \mathcal{F}_{t}\right\}
$$


represents the total wealth for the replicating strategy and the price at time $t$ of a zero-coupon bond with the payoff $\$ 1$ at the maturity time $T$.

Let us discuss some consequences of these statements.

Lemma 4.1 implies that the value

$$
r(t) \triangleq-(T-t)^{-1} \log P(t, T)=-(T-t)^{-1} \log \left(B(t) \mathbf{E}_{\theta}\left\{B(T)^{-1} \mid \mathcal{F}_{t}\right\}\right)
$$

represents the so-called yield to maturity, or the expected average risk-free rate associated with the zero-coupon bond, meaning that the price at time $t$ of a zero-coupon bond with the payoff $\$ 1$ at the maturity time $T$ is

$$
P(t, T)=\exp (-r(t)(T-t))
$$

If the processes $a(t), \sigma(t), \alpha(t), \rho(t), \widehat{\rho}(t), \theta(t)$ are constant and the assumptions of Lemma 4.1 are satisfied, then

$$
B(T)^{-1}=B(t)^{-1} \exp \left(\left(-\alpha+\frac{\rho^{2}}{2}+\rho \theta_{1}\right)[T-t]-\rho\left(W_{1 \theta}(T)-W_{1 \theta}(t)\right)\right) .
$$

In this case, a direct calculation of (4.1) gives

$$
-(T-t)^{-1} \log P(t, T)=\alpha-\rho^{2}-\rho \theta_{1} .
$$

Since $\theta_{1}=\widetilde{a} / \widetilde{\sigma}$, it gives that $-(T-t)^{-1} \log P(t, T)=\alpha-\rho^{2}-\rho \widetilde{a} / \widetilde{\sigma}$. Hence

$$
r=\alpha-\rho^{2}-\rho \widetilde{a} / \widetilde{\sigma}
$$

can be interpreted as the "true" risk-free rate for this market. It can be seen that $r$ is close to $\alpha$ if $\rho$ is small; if $\rho=0$ then $r=\alpha$.

Further, let us consider a scenario where the real market price $P_{\text {market }}(0, T)$ of a zerocoupon bond with the payoff $\$ 1$ at the maturity time $T$ is observed from the market statistics at time $t=0$, and the corresponding value (4.1) $r_{\text {market }}=-T^{-1} \log P_{\text {market }}(0, T)$ is calculated. If $\rho=0$ then only $r_{\text {market }}=\alpha$ is consistent with (4.1). Assume that $\rho \neq 0$. In this case, we can reverse pricing formula (4.1) and calculate implied $\theta_{1, \text { implied }}$ from (4.5) as

$$
\theta_{1, \text { implied }}=\left(\alpha-\rho^{2}-r_{\text {market }}\right) / \rho \text {. }
$$


In this case, equation (2.1) can be rewritten as

$$
d B(t)=B(t)\left(\left[r_{\text {market }}+\rho^{2}\right] d t+\rho d W_{1 \theta}(t)\right)
$$

where $W_{1 \theta}(t)=w(t)+\int_{0}^{t} \theta_{1, \text { implied }}(s) d s$.

It particular, it follows that a choice of $\rho$ for a market model with given $\alpha$ is consistent with the observed bond prices if $\left(\alpha-r_{\text {market }}\right) / \rho$ is bounded as $\rho \rightarrow 0$. This leads to the following heuristic rule: if the observed bond market price is such that $\alpha-r_{\text {market }}$ is large, then one should assume a sufficiently large $\rho$, to avoid overestimation of the market price of risk.

Representation (4.6) follows the classical approach to the so-called implied volatility where the Black-Scholes formula is reversed. However, there is some novelty: as far as we know, this is the first attempt to derive the implied market price of risk process generated by the appreciation rate of a stock prices. Currently, there are few other implied processes considered in the literature, besides the classical implied volatility. Turvey and Komar (2006) considered inference of the implied value $a-\theta_{1} \sigma$ from the market option price, in a model that corresponds to our model with $\widehat{\sigma}=\rho=\widehat{\rho}=0$, presuming that this value is used as the appreciation rate under the pricing measure; for the Black and Scholes model, this value should be the risk-free rate. The implied cumulate risk-free rate was considered in the framework of Black and Scholes model in Dokuchaev (2006) and Hin and Dokuchaev (2014), as an inferred parameters from stock option prices. Weron (2008) estimated the implied market price of risk for energy prices as the difference between the observable historical Ornstein-Uhlenbeck long term mean and the implied long-term mean infrared from the market options prices. Finally, the implied martingale measure for a bond market was introduced in Bielecki et al. (2009); this construction was based on observation of bond prices for a continuum of maturities. None of these papers considered estimation of the appreciation rate of the stock as an implied parameter inferred from the stock option prices. The implied martingale measure defined by (4.6) has a different nature: it is associated with a market price of risk process $\widetilde{a}(t) / \widetilde{\sigma}(t)$ and the appreciation rate $a(t)$ of the stock prices.

It appears that estimation of the appreciation rate of stock prices and the market price of risk process $\theta(t)$ from the historical data is a quite challenging problem that is important for financial applications, especially for optimal portfolio selection. For financial models, 
estimation of these processes is more difficult than estimation of the volatility since the trend for financial time series is usually relatively small and unstable. Some results and references for the estimation of the appreciation rate and application to portfolio selection can be found in Brennan (1998), Dokuchaev (2005), and Dokuchaev (2002), Ch.9, p.128. Calculation of the implied $\theta$ form observed bond prices as described above could be a useful addition to the existing methods. Further development of this approach is presented in Section 7 below.

Remark 4.1 For the bond pricing model with constant coefficients described above, the choice of $\left(\theta_{1}, r\right)$ is independent on $T$. It follows that a single market price $P_{\text {market }}(0, T)$ of a zero coupon bond for one given maturity time $T$ defines uniquely the prices of similar bonds for all other maturity times $\bar{T} \neq T$ given that these prices are defined by 4.1). This is caused by the fact that this formula has to be applied with the same $\theta_{1}$ leading to the same $r$ in 4.3). It can be noted that, for models with time variable coefficients of equations for $(B, S)$, the same approach gives a time dependent $\left(\theta_{1}(t), r(t)\right)$, and the value 4.5) defined for a maturity time $\bar{T}$ depends on $\bar{T}$.

Remark 4.2 Under the assumptions of Theorems [3.1] 3.2, the choice of $\gamma$ is unique, i.e., it is the same for all $\theta \in \mathcal{T}$ such that $\mathbf{E}_{\theta} \xi^{2}<+\infty$; the expectation $\mathbf{E}_{\theta} \xi$ is also the same for all these $\theta$.

\section{On relativity of the price and the hedging error}

The number $c_{\theta}=\mathbf{E}_{\theta} \xi$ is commonly regarded as the price of an option with the payoff $B(T) \xi$. This price depends on the selection of $\theta$. The following theorems demonstrate that this price can be selected quite arbitrarily even for the case of an arbitrarily small stochastic deviations in (2.1) , i.e., for arbitrarily small processes $\rho(t)$ and $\widehat{\rho}(t)$. For instance, we can select $\rho(t) \equiv \widehat{\rho}(t)=\varepsilon$ for an arbitrarily small $\varepsilon>0$. This means that the presence of small deviations in (2.1) changes dramatically the properties of the market model.

We denote $x^{+}=\max (0, x)$ for $x \in \mathbf{R}$.

Theorem 5.1 Assume that

$$
\underset{t, \omega}{\operatorname{essinf}}|\widetilde{\sigma}(t, \omega) \widehat{\rho}(t, \omega)-\rho(t, \omega) \widetilde{\rho}(t, \omega)|>0 .
$$


Let $\kappa \in(0,+\infty)$ be given, and let $\xi=B(T)^{-1}(\kappa-S(T))^{+}$. Then the following holds.

(i) for any $\varepsilon>0$, there exists $\theta \in \mathcal{T}$ such that $c_{\theta}=\mathbf{E}_{\theta} \xi \in[0, \varepsilon]$, and

(ii) For any $M>0$, there exists $\theta \in \mathcal{T}$ such that $c_{\theta}=\mathbf{E}_{\theta} \xi \geq M$.

Theorem 5.2 Assume that (5.1) holds. Let $\kappa \in(0,+\infty)$ be given, and let $\xi=B(T)^{-1}(S(T)-$ $\kappa)^{+}$. Then the following holds.

(i) For any $\varepsilon>0$, there exists $\theta \in \mathcal{T}$ such that $c_{\theta}=\mathbf{E}_{\theta} \xi \in[0, \varepsilon]$, and

(ii) For any $\varepsilon>0$, there exists $\theta \in \mathcal{T}$ such that $c_{\theta}=\mathbf{E}_{\theta} \xi \in[S(0)-\varepsilon, S(0)]$.

Consider a hedging strategy that replicates the claim $B(T)\left(c_{\theta}+I_{\theta}\right)$, where $c_{\theta} \in \mathbf{R}$ and $I_{\theta} \in \mathcal{X}_{\theta}$ are such that (3.2) holds with some $R_{\theta} \in \mathcal{X}_{\theta}^{\perp}$. This $R_{\theta}$ is the hedging error.

The following theorems show that the value of the second moment of $R_{\theta}$ is varying widely with variations of the historical measure and take can extreme values for some choices of the equivalent martingale measures.

Theorem 5.3 Let $\xi$ be a random claim such that (3.2) holds for some $\theta \in \mathcal{T}, c_{\theta} \in \mathbf{R}$, $I_{\theta} \in \mathcal{X}_{\theta}$, and $R_{\theta} \in \mathcal{X}_{\theta}^{\perp}$ such that $\mathbf{E}_{\theta} R_{\theta}^{2}>0$. Assume that (3.1) holds for $U_{\theta} \in \mathcal{Y}_{\theta}$ such that

$$
\underset{\omega}{\operatorname{ess} \sup } \int_{0}^{T}\left|U_{\theta}(t, \omega)\right|^{2} d t<+\infty, \quad \underset{\omega}{\operatorname{essinf}} \int_{0}^{T}\left|\eta_{\theta}(t, \omega)\right| d t>0
$$

for the process $\eta_{\theta}$ defined by 3.4). Then the following holds:

(i) For any $M>0, \mathbf{P}\left(R_{\theta}^{2}>M\right)>0$;

(ii) For any $M>0$, there exists a measure $Q$ that is equivalent to $\mathbf{P}$ and such that $\mathbf{E}_{Q} R_{\theta}^{2} \geq$ $M$, where $\mathbf{E}_{Q}$ is the corresponding expectation.

Theorem 5.4 Let $\xi$ be a random claim such that (3.2) holds for some $\theta \in \mathcal{T}, c_{\theta} \in \mathbf{R}$, $I_{\theta} \in \mathcal{X}_{\theta}$, and $R_{\theta} \in \mathcal{X}_{\theta}^{\perp}$ such that $\mathbf{E}_{\theta} R_{\theta}^{2}>0$. Assume that (3.1) holds for $U_{\theta} \in \mathcal{Y}_{\theta}$ such that

$$
\underset{t, \omega}{\operatorname{ess} \sup }\left|U_{\theta}(t, \omega)\right|<+\infty, \quad \quad \underset{t, \omega}{\operatorname{essinf}}\left|\eta_{\theta}(t, \omega)\right|>0
$$

for the process $\eta_{\theta}$ defined by 3.4). Then 
(i) For any $\varepsilon>0, \mathbf{P}\left(R_{\theta}^{2}<\varepsilon\right)>0$;

(ii) for any $\varepsilon>0$, there exists a measure $Q$ that is equivalent to $\mathbf{P}$ and such that $\mathbf{E}_{Q} R_{\theta}^{2} \leq \varepsilon$, where $\mathbf{E}_{Q}$ is the corresponding expectation.

Remark 5.1 Clearly, the statement of Theorem 5.3 holds if and only if $\mathbf{P}\left(R_{\theta}^{2}>M\right)>0$ for any $M>0$, and the statement of Theorem 5.4 holds if and only if $\mathbf{P}\left(R_{\theta}^{2}<\varepsilon\right)>0$ for any $\varepsilon>0$. Therefore, the statements of Theorems 5.35 .4 can be reformulated as the following: the assumptions imposed there on $U$ and $\eta_{\theta}$ imply the corresponding conditions (i).

\section{On selection of $\theta$ and the equivalent martingale measure}

Since the equivalent martingale measure is not unique, a question arises which particular $\theta$ should be used for calculation of the price $c_{\theta}=\mathbf{E}_{\theta} \xi$. In the literature, there are many methods developed for this problem, mainly for the incomplete market models with random volatility and appreciation rate.

One may look for "optimal" $\theta$ and $c_{\theta}$ in the spirit of mean-variance pricing, such that $\mathbf{E} R_{\theta}^{2}$ is minimal; see, e.g., Schweizer (2001). A generalization of this approach leads to minimization of $\mathbf{E}\left|R_{\theta}\right|^{q}$ for $q \geq 1$. An alternative approach is to define the price as $\sup _{\theta \in \mathcal{T}_{0}} c_{\theta}$ for some reasonably selected set $\mathcal{T}_{0} \subset \mathcal{T}$. In the case of an incomplete market with random volatility, this pricing rule leads to a corrected volatility smile (Dokuchaev (2011)).

The following Theorem 6.1 will be useful for selection of $\theta$.

Theorem 6.1 Let $\theta=\left(\theta_{1}, \theta_{2}\right)^{\top} \in \mathcal{T}$ be given, and let $\varrho(t)=\widehat{V}(t)^{\top} \theta(t)$, i.e.,

$$
\begin{aligned}
\widetilde{\sigma} \theta_{1}+\widetilde{\rho} \theta_{2} & =\widetilde{a}, \\
\rho \theta_{1}+\widehat{\rho} \theta_{2} & =\varrho .
\end{aligned}
$$

Then

$$
\begin{aligned}
& d S(t)=S(t)\left([a(t)-\widetilde{a}(t)-\varrho(t)] d t+\sigma(t) d W_{1 \theta}(t)+\widehat{\sigma}(t) d W_{2 \theta}(t)\right), \\
& \left.d B(t)=B(t)\left([\alpha(t)-\varrho(t)] d t+\rho(t) d W_{1 \theta}(t)\right)+\widehat{\rho}(t) d W_{2 \theta}(t)\right) .
\end{aligned}
$$

Clearly, if (5.1) holds, then a unique $\theta$ can be found from system (6.1) for any $\mathbb{F}$-adapted and bounded process $\varrho(t)$. In this case, Theorem 6.1 gives a useful parametrization of the set 
$\mathcal{T}$ via $\varrho$. Examples 6.1 6.4 below demonstrate how parametrization of $\mathcal{T}$ via $\varrho$ helps to find some reasonable choices of $\theta$. For these examples, we assume that (5.1) holds.

Example 6.1 For $\theta$ from Theorem 6.1 with $\varrho \equiv 0$, the process $(S(t), B(t))$ evolves as

$$
\begin{aligned}
& d S(t)=S(t)\left([a(t)-\widetilde{a}(t)] d t+\sigma(t) d W_{1 \theta}(t)+\widehat{\sigma}(t) d W_{2 \theta}(t)\right) \\
& \left.d B(t)=B(t)\left(\alpha(t) d t+\rho(t) d W_{1 \theta}(t)\right)+\widehat{\rho}(t) d W_{2 \theta}(t)\right)
\end{aligned}
$$

In particular, the equation for $B$ has the same coefficients as the equation for $B(t)$ under $\mathbf{P}$, with replacement of $W(t)$ by $W_{\theta}(t)$. In particular, the distribution of $B(t)$ under $\mathbf{P}_{\theta}$ and under the historical measure $\mathbf{P}$ is the same if the coefficients $\alpha(t), \rho(t)$, and $\widehat{\rho}(t)$, are non-random. In addition, if $\widehat{\sigma} \equiv 0$ then the choice $\varrho \equiv 0$ ensures that $\theta_{1}=\widehat{a} / \sigma$.

Example 6.2 For $\theta$ from Theorem 6.1 with $\varrho=-\widetilde{a}$, the evolution of $S$ under $\mathbf{P}_{\theta}$ is described by an Itô equation with the same coefficients as the equation for $S(t)$ under $\mathbf{P}$, with replacement of $W(t)$ by $W_{\theta}(t)$. The distribution of $S(t)$ under $\mathbf{P}_{\theta}$ and under the historical measure $\mathbf{P}$ is the same for the case of non-random coefficients $a(t), \sigma(t)$, and $\widehat{\sigma}(t)$.

Example 6.3 Let $k \in(0,1)$ be given. Let us calculate $\theta$ from (6.1) with $\varrho=\frac{k}{1-k}\left(\rho^{2}+\widehat{\rho}^{2}\right)$, i.e.,

$$
\begin{aligned}
& \widetilde{\sigma} \theta_{1}+\widetilde{\rho} \theta_{2}=\widetilde{a}, \\
& \rho \theta_{1}+\widehat{\rho} \theta_{2}=\frac{k}{1-k}\left(\rho^{2}+\widehat{\rho}^{2}\right) .
\end{aligned}
$$

The second equation here can be rewritten as

$$
\rho \theta_{1}+\widehat{\rho} \theta_{2}=k\left(\rho^{2}+\widehat{\rho}^{2}+\rho \theta_{1}+\widehat{\rho} \theta_{2}\right) .
$$

Let $r_{\theta}$ be defined by (7.2). This implies that $k\left(\rho^{2}+\widehat{\rho}^{2}+\rho \theta_{1}+\widehat{\rho} \theta_{2}\right)=k\left(\alpha-r_{\theta}\right)$. It follows that $\theta$ satisfies system (6.1) with $\varrho=\varrho(\theta)=k\left(\alpha-r_{\theta}\right)$. By (6.2), this leads to the equation

$$
\left.d B(t)=B(t)\left(\left[k r_{\theta}+(1-k) \alpha\right] d t+\rho d W_{1 \theta}(t)\right)+\widehat{\rho} d W_{2 \theta}(t)\right)
$$

i.e., the appreciation rate coefficient for $B$ under $\mathbf{P}_{\theta}$ is $k r_{\theta}+(1-k) \alpha$. Therefore, we have established that there exists a choice of $\theta$ that ensures that the appreciation rate for $B$ under $\mathbf{P}_{\theta}$ can be arbitrarily close to $r_{\theta}$ representing the expected average risk-free rate associated with the zero-coupon bond under the measure $\mathbf{P}_{\theta}$. This can be achieved with selection of $k$ close to 1 . 
Example 6.4 An important example of the selection of $\theta$ is

$$
\theta(t)=\widetilde{a}(t) V(t) /|V(t)|^{2}
$$

The following theorem shows that this corresponds to the choice of $\theta$ with the minimal norm.

Theorem 6.2 Let $\theta(t)$ be defined by (6.3). Then, for every $t, \omega$, the value of $|\theta(t, \omega)|$ is minimal among all $\theta \in \mathcal{T}$. In addition, if $\xi=c_{\theta}+\int_{0}^{T} \gamma_{\theta}(t) d \widetilde{S}(t)+R_{\theta}$ for some $R_{\theta} \in \mathcal{X}_{\theta}^{\top}$ and $\gamma_{\theta}$ is an adapted process such that $\gamma_{\theta} \sigma \in \mathcal{H}_{\theta}$, then $\mathbf{E}\left(R_{\theta} \mathcal{M}(T)\right)=0$, where $\mathcal{M}(T)=$ $\int_{0}^{T} \gamma_{\theta}(t) \widetilde{S}(t) V(t)^{\top} d W(t)$ represents the "martingale" part of the integral

$$
\int_{0}^{T} \gamma_{\theta}(t) d \widetilde{S}(t)=\int_{0}^{T} \gamma_{\theta}(t) \widetilde{S}(t) \widehat{a}(t) d t+\mathcal{M}(T) .
$$

The selection of $\theta$ described in Theorem 6.2 ensures that the corresponding self-financing strategy with the quantity of shares $\gamma(t)$ is a so-called locally risk minimizing strategy; see, e.g., Föllmer and Sondermann (1986), Biagini and Pratelli (1999).

Let us reconsider Example 2.1 (iv). We will be using the measure $\widehat{\mathbf{P}}$ and the processes $q(t), z(t)$, and $\widehat{z}(t)$ defined in this example.

Set $\mathcal{V}(t)=\widehat{V}(t)-k(t) V$, where

$$
k(t)=\widehat{V}(t)^{\top} V(t) /|V(t)|^{2} .
$$

Clearly, we have that $\mathcal{V}(t)^{\top} V(t)=0$.

Further, there exists a one-dimensional Wiener process $z_{1}(t)$ such that $\int_{0}^{t} \mathcal{V}(s)^{\top} d W(s)=$ $\int_{0}^{t}|\mathcal{V}(s)| d z_{1}(s)$ and

$$
\begin{aligned}
d B(t) & =B(t)\left(\alpha(t) d t+k(t) V(t)^{\top} d W(t)+\mathcal{V}(t)^{\top} d W(t)\right) \\
& =B(t)\left(\alpha(t) d t+k(t)|V(t)| d z(t)+|\mathcal{V}(t)| d z_{1}(t)\right)
\end{aligned}
$$

For $q(t)=\widetilde{a}(t) /|V(t)|$, we have

$$
d B(t)=B(t)\left(\alpha(t) d t+k(t)|V(t)|(d \widehat{z}(t)-q(t) d t)+|\mathcal{V}(t)| d z_{1}(t)\right)
$$

On the other hand,

$$
d B(t)=B(t)\left(\alpha(t) d t+\widehat{V}(t)^{\top} d W(t)\right)=B(t)\left(\alpha(t) d t+\widehat{V}(t)^{\top}\left(d W_{\theta}(t)-\theta(t) d t\right)\right.
$$


This means that, in our notation, $\widehat{\mathbf{P}}=\mathbf{P}_{\theta}$, where $\theta \in \mathcal{T}$ is such that

$$
k(t) q(t)|V(t)|=k(t) \widetilde{a}(t)=\widehat{V}(t)^{\top} \theta(t) .
$$

The only $\theta \in \mathcal{T}$ satisfying this is $\theta(t)=\widetilde{a}(t) V(t) /|V(t)|^{2}$ from Theorem 6.2,

\section{The implied market price of risk for an incomplete market}

In Section 4, we established that the value (4.1) represents the price at time $t$ of a zero-coupon bond with the payoff $\$ 1$ at the maturity time $T$ for a case where the claim $\$ 1$ is replicable. This implies that it could be reasonable to accept (4.1) as the price of this bond for a more general case of a non-replicable claim $\$ 1$ as well (i.e., with non-zero $\rho$ and $\widehat{\rho}$ ). This means that $r(t)$ defined by (4.2) represents again the expected average risk-free rate associated with the zero-coupon bond.

This would require to produce on more example of selection of $\theta$.

Assume that the processes $a(t), \sigma(t), \alpha(t), \rho(t), \widehat{\rho}(t), \theta(t)$ are constant, and that $r(t)$ is defined by (4.2). It follows from Itô formula that

$$
\begin{aligned}
B(T)^{-1}= & B(t)^{-1} \exp \left(\left(-\alpha+\frac{\rho^{2}}{2}+\frac{\widehat{\rho}^{2}}{2}+\rho \theta_{1}+\widehat{\rho} \theta_{2}\right)(T-t)\right. \\
& \left.-\rho\left(W_{1 \theta}(T)-W_{1 \theta}(t)\right)-\widehat{\rho}\left(W_{2 \theta}(T)-W_{2 \theta}(t)\right)\right) .
\end{aligned}
$$

Then (4.2) imply that $r(t)$ can be found explicitly; it does not depend on $t$ and $T$ and depend on $\theta$ such that $r(t) \equiv r_{\theta}$, where

$$
r_{\theta}=\alpha-\rho^{2}-\widehat{\rho}^{2}-\rho \theta_{1}-\widehat{\rho} \theta_{2}
$$

Equation (7.2) can be rewritten as

$$
\rho \theta_{1}-\widehat{\rho} \theta_{2}=\alpha-r_{\theta}-\rho^{2}-\widehat{\rho}^{2}
$$

Following the approach from Section 4, consider now a scenario where the real market price $P_{\text {market }}(0, T)$ of a zero-coupon bond with the payoff $\$ 1$ at the maturity time $T$ is observed from the market statistics at time $t=0$, and the corresponding value (4.1) 
$r_{\text {market }}=-T^{-1} \log P_{\text {market }}(0, T)$ is calculated. We can reverse pricing formula (4.1) and calculate implied $\theta=\theta_{\text {implied }}$ as solution of system (6.1) with

$$
\varrho=\alpha-r_{\text {market }}-\rho^{2}-\widehat{\rho}^{2} .
$$

This would follow again the classical approach to the so-called implied volatility where the Black-Scholes formula is reversed.

In particular, equation (6.2) for this $\varrho$ implies that

$$
\left.d B(t)=B(t)\left(\left[r_{\text {market }}+\rho^{2}+\widehat{\rho}^{2}\right] d t+\rho d W_{1 \theta}(t)\right)+\widehat{\rho} d W_{2 \theta}(t)\right)
$$

where $\theta=\theta_{\text {implied }}$.

This model has the same feature as described in Remark 4.1 for a special model. In particular, the choice of $\theta$ is independent on $T$, and a single market price $P_{\text {market }}(0, T)$ of a zero coupon bond for one given maturity time $T$ defines uniquely the prices defined by (4.1) for similar bonds for all other maturity times $\bar{T} \neq T$, since this formula has to be applied with the same $\theta$. For models with time variable coefficients of equations for $B$ and $S$, the same approach gives a time dependent solution $\theta(t)$ of (6.1), and the value $r$ defined by (4.5) for a maturity time $\bar{T}$ depends on $\bar{T}$.

\section{Markov case}

In this section, we suggests some representations of prices, errors, and hedging strategies via solutions of deterministic PDEs.

We will be using the processes $\mathrm{s}(t)=\log \widetilde{S}(t)$ and $\mathrm{b}(t)=\log B(t)$. By Itô formula, we obtain that

$$
\begin{aligned}
d \mathrm{~s}(t) & =\left(\widetilde{a}-\widetilde{\sigma}^{2} / 2-\widetilde{\rho}^{2} / 2\right) d t+\widetilde{\sigma} d w(t)+\widetilde{\rho} d \widehat{w}(t), \\
d \mathrm{~b}(t) & =\left(r-\rho^{2} / 2-\widehat{\rho}^{2} / 2\right) d t+\rho d w(t)+\widehat{\rho} d \widehat{w}(t)
\end{aligned}
$$

Assume that $\theta \in \mathcal{T}$ is given. In this section, we will assume that there exists a measurable function $f: \mathbf{R}^{2} \times[0, T] \rightarrow \mathbf{R}^{8}$ such that

$$
\left(\widetilde{a}(t), \widetilde{\sigma}(t), \widetilde{\rho}(t), \alpha(t), \rho(t), \widehat{\rho}(t), \theta_{1}(t), \theta_{2}(t)\right)^{\top}=f(\mathrm{~s}(t), \mathrm{b}(t), t)
$$


To simplify notation, we will describe it as the following: we assume that there are measurable functions $\widetilde{a}(s, b, t), \widetilde{\sigma}(s, b, t), \widetilde{\rho}(s, b, t), \alpha(s, b, t), \rho(s, b, t), \widehat{\rho}(s, b, t), \theta(s, b, t)$ defined on $\mathbf{R}^{2} \times$ $[0, T]$ and such that the processes $\widetilde{a}(t), \widetilde{\sigma}(t), \widetilde{\rho}(t), \alpha(t), \rho(t), \widehat{\rho}(t), \theta(t)$ (defined on $[0, T] \times$ $\Omega$ ) are replaced by the processes $\widetilde{a}(\mathrm{~s}(t), \mathrm{b}(t), t), \widetilde{\sigma}(\mathrm{s}(t), \mathrm{b}(t), t), \widetilde{\rho}(\mathrm{s}(t), \mathrm{b}(t), t), \alpha(\mathrm{s}(t), \mathrm{b}(t), t)$, $\rho(\mathrm{s}(t), \mathrm{b}(t), t), \widehat{\rho}(\mathrm{s}(t), \mathrm{b}(t), t)$, and $\theta(\mathrm{s}(t), \mathrm{b}(t), t)$, respectively.

Let $\xi=F(\widetilde{S}(T), B(T))$, where $F:(0,+\infty)^{2} \rightarrow \mathbf{R}$ is a measurable function such that $\mathbf{E}_{\theta} \xi^{2}<+\infty$ for some $\theta \in \mathcal{T}$. Consider the pricing and hedging problem for the claim $B(T) \xi$. Let us calculate the price $c_{\theta}=\mathbf{E}_{\theta} \xi$, and the hedging strategy $\gamma(t)$ in (3.2) and (3.5).

Let $H=H_{\theta}=H_{\theta}(s, b, t)$ be the solution of the following linear parabolic equation in $\mathbf{R}^{2} \times[0, T]:$

$$
\begin{aligned}
& H_{t}^{\prime}+H_{s}^{\prime}\left(\widetilde{a}-\widetilde{\sigma}^{2} / 2-\widetilde{\rho}^{2} / 2\right)+H_{b}^{\prime}\left(\alpha-\rho^{2} / 2-\widehat{\rho}^{2} / 2\right)+\mathcal{L} H=H_{s}^{\prime} \theta_{1}+H_{b}^{\prime} \theta_{2}, \\
& H(s, b, T)=F\left(e^{s}, e^{b}\right) .
\end{aligned}
$$

Here

$$
\mathcal{L} H=\frac{1}{2}\left(\begin{array}{c}
\widetilde{\sigma} \\
\rho
\end{array}\right)^{\top} H^{\prime \prime}\left(\begin{array}{c}
\widetilde{\sigma} \\
\rho
\end{array}\right)+\frac{1}{2}\left(\begin{array}{c}
\widetilde{\rho} \\
\widehat{\rho}
\end{array}\right)^{\top} H^{\prime \prime}\left(\begin{array}{c}
\widetilde{\rho} \\
\widehat{\rho}
\end{array}\right), \quad H^{\prime \prime}=\left(\begin{array}{cc}
H_{s s}^{\prime \prime} & H_{s b}^{\prime \prime} \\
H_{b s}^{\prime \prime} & H_{b b}^{\prime \prime}
\end{array}\right) .
$$

Assume that there exists a generalized solution $H(s, b, t)$ of (8.2) such that its gradient with respect to $(s, b)$ is bounded. By Itô formula, it follows that (3.1) holds with

$$
U_{\theta}(t)=H_{s}^{\prime}(\mathrm{s}(t), \mathrm{b}(t), t) V(t)+H_{b}^{\prime}(\mathrm{s}(t), \mathrm{b}(t), t) \widehat{V}(t)
$$

In this case, (3.2) and (3.5) hold with

$$
c_{\theta}=H(\log S(0), \log B(0), 0), \quad \gamma(t)=\nu_{\theta}(t) \widetilde{S}(t)^{-1},
$$

where $\nu_{\theta}(t)=\frac{U_{\theta}^{\top} V}{|V|^{2}}=f_{\theta}(\mathrm{s}(t), \mathrm{b}(t), t)$, and where

$$
f_{\theta}(s, b, t)=H_{s}^{\prime}(\mathrm{s}(t), \mathrm{b}(t), t)+\frac{H_{b}^{\prime}(\mathrm{s}(t), \mathrm{b}(t), t)(\widetilde{\sigma}(s, b, t) \rho(s, b, t)+\widetilde{\rho}(s, b, t) \widehat{\rho}(s, b, t))}{\widetilde{\sigma}(s, b, t)^{2}+\widetilde{\rho}(s, b, t)^{2}} .
$$

Further, let us consider the problem of calculation of $\mathbf{E} R_{\theta}^{2}$, i.e., estimation of the hedging error with respect to the historical measure $\mathbf{P}$. We have that (3.2)-(3.5) hold with $\eta_{\theta}(t)=$ 
$U_{\theta}(t)-\nu_{\theta}(t) V(t)=g_{\theta}(\mathrm{s}(t), \mathrm{b}(t), t)$, where

$$
g_{\theta}(s, b, t)=H_{s}^{\prime}(s, b, t) V(t)+H_{b}^{\prime}(s, b, t) \widehat{V}(t)-f_{\theta}(s, b, t)\left(\begin{array}{c}
\widetilde{\sigma}(s, b, t) \\
\widetilde{\rho}(s, b, t)
\end{array}\right) .
$$

Let

$$
A(s, b, t)=g_{\theta}(s, b, t)^{\top} \theta(s, b, t), \quad v(s, b, t)=g_{\theta}(s, b, t) .
$$

It can be noted that, under the assumption of Proposition 3.1. $R_{\theta}=x(T)$, where the process $x(t)$ evolves as

$$
d x(t)=\eta_{\theta}(t)^{\top} \theta(t) d t+\eta_{\theta}(t)^{\top} d W(t), \quad x(0)=0 .
$$

By $(\underline{8.3)}), \mathbf{E} R_{\theta}^{2}=\mathbf{E} x(T)^{2}$, where

$$
d x(t)=A(\mathrm{~s}(t), \mathrm{b}(t), t) d t+v(\mathrm{~s}(t), \mathrm{b}(t), t)^{\top} d W(t) .
$$

This equation together with (8.1) describes evolution of a diffusion Markov process $(x(t), \mathrm{s}(t), \mathrm{b}(t))$. Therefore,

$$
\mathbf{E} R_{\theta}^{2}=\mathbf{E} x(T)^{2}=J(0, \log S(0), \log B(0), 0),
$$

where $J(x, s, b, t)$ is the solution of the corresponding backward Kolmogorov-Fokker-Planck parabolic equation for $(x(t), \mathrm{s}(t), \mathrm{b}(t))$ in $\mathbf{R}^{3} \times[0, T]$

$$
\begin{aligned}
& J_{t}^{\prime}+J_{x}^{\prime} A+J_{s}^{\prime}\left(\widetilde{a}-\widetilde{\sigma}^{2} / 2-\widetilde{\rho}^{2} / 2\right)+J_{b}^{\prime}\left(r-\rho^{2} / 2-\widehat{\rho}^{2} / 2\right)+\mathcal{D} J \\
& J(x, y, z, T)=x^{2}
\end{aligned}
$$

Here

$\mathcal{D} J=\frac{1}{2}\left(\begin{array}{c}v_{1} \\ \widetilde{\sigma} \\ \rho\end{array}\right)^{\top} J^{\prime \prime}\left(\begin{array}{c}v_{1} \\ \widetilde{\sigma} \\ \rho\end{array}\right)+\frac{1}{2}\left(\begin{array}{c}v_{2} \\ \widetilde{\rho} \\ \widehat{\rho}\end{array}\right)^{\top} J^{\prime \prime}\left(\begin{array}{c}v_{2} \\ \widetilde{\rho} \\ \widehat{\rho}\end{array}\right), \quad J^{\prime \prime}=\left(\begin{array}{ccc}J_{x x}^{\prime \prime} & J_{x y}^{\prime \prime} & J_{x z}^{\prime \prime} \\ J_{y x}^{\prime \prime} & J_{y y}^{\prime \prime} & J_{y z}^{\prime \prime} \\ J_{z x}^{\prime \prime} & J_{z y}^{\prime \prime} & J_{z z}^{\prime \prime}\end{array}\right)$. 


\section{Conclusions}

We revisited the problem of pricing of stock options for the case of the market model with a stochastic numéraire, with emphasize on the impact of the non-uniqueness of equivalent martingale measures and the presence of non-replicable claims. We found that there are some interesting features that makes this model different from incomplete market models where incompleteness is caused by the randomness of the volatility. We found that the martingale prices vary significantly for some extreme choices of the equivalent martingale measures. For instance, for a European put option, any sufficiently large positive real number is a martingale price for some equivalent martingale measure. Some possible economically justified choices of equivalent martingale measures are suggested, including a measure that correspond to a consensus about the future numéraire process, a measure that ensures local risk minimizing hedging strategy, and an implied measure that takes into account observed zero coupon bond prices. This last measure is especially interesting, since it leads to a formula for an implied market price of risk.

It could be interesting to consider optimal selection of the equivalent martingale measure in the spirit of the mean-variance hedging. It also could be interesting to develop a comprehensive bond pricing model that is based on stochastic numéraire with time dependent coefficients, and investigate dependence of the price on the maturity time. We leave it for future research.

\section{Appendix: Proofs}

Proof of Lemma 2.1 is straightforward and based on the application of Itô's formula. In fact, Lemma 2.1 represents a special case of Proposition 1 from Geman et al. (1995).

Proof of Lemma 2.2 follows immediately from equation (2.11) and from the fact that $d \widetilde{S}(t)=\widetilde{S}(t) V(t)^{\top} d W_{\theta}(t)$.

Proof of Proposition 3.2. By Lemma 2.1 and 2.2, the set $\mathcal{X}_{\theta}$ contains random variables

$$
\int_{0}^{T} \gamma(t) d \widetilde{S}(t)=\int_{0}^{T} \gamma(t) \widetilde{S}(t) V(t)^{\top} d W_{\theta}(t)
$$

where $\gamma \in \mathcal{H}_{\theta}$ and where $W_{\theta}$ is defined by (2.6). 
For any $\zeta \in \mathcal{X}_{\theta}^{\perp}$, there exists $U(t)=\left(U_{1}(t), U_{2}(t)\right)^{\top} \in \mathcal{Y}_{\theta}$ such that

$$
\zeta=\int_{0}^{T} U(t)^{\top} d W_{\theta}(t)
$$

Let us show that if $\zeta \in \mathcal{X}_{\bar{\theta}}^{\perp}$ then $U(t)^{\top} V(t)=0$. For this $\zeta$, we have that

$$
\mathbf{E}_{\theta} \zeta \int_{0}^{T} \gamma(t) d \widetilde{S}(t)=\mathbf{E}_{\theta} \int_{0}^{T} \gamma(t) \widetilde{S}(t) V(t)^{\top} U(t) d t=0 \quad \forall \gamma \in \mathcal{H}_{\theta} .
$$

Hence $\widetilde{S}(t) V(t)^{\top} U(t)=0$ a.e. Hence $V(t)^{\top} U(t)=0$ a.e.

To show that the set $\mathcal{X}_{\theta}^{\perp}$ contains non-zero elements, it suffices to take $U_{1}(t)=\psi(t) \widehat{\rho}(t)$ and $U_{2}(t)=\psi(t) \widetilde{\sigma}(t)$, with an arbitrary $\psi \in \mathcal{Y}_{\theta}$, i.e.,

$$
\zeta=\int_{0}^{T} \psi(t)\left[\widehat{\rho}(t) d W_{\theta 1}(t)+\widetilde{\sigma}(t) d W_{\theta 2}(t)\right] .
$$

This completes the proof.

Proof of Theorem 3.1. Under the assumptions, $d \widetilde{S}(t)=\widetilde{S}(t)|V(t)| d z_{\theta}(t)$, where $z_{\theta}(t)$ is a one-dimensional Wiener process such that $\int_{0}^{t} V(s)^{\top} d W_{\theta}(s)=\int_{0}^{t}|V(s)| d z_{\theta}(s)$. Hence the filtration $\left(\mathcal{F}_{t}^{z_{\theta}}\right)_{t \geq o}$ generated by $z_{\theta}(t)$ is such that $\mathcal{F}_{T}^{z_{\theta}}=\mathcal{F}_{T}^{\widetilde{S}}$. Hence any $\xi \in L_{2}\left(\Omega, \mathcal{F}_{T}^{\widetilde{S}}, \mathbf{P}_{\theta}\right)$ belongs to $L_{2}\left(\Omega, \mathcal{F}_{T}^{z_{\theta}}, \mathbf{P}_{\theta}\right)$. By the Martingale Representation Theorem, it follows that there exists an $\mathcal{F}_{t}^{z_{\theta}}$-adapted process $u_{\theta}(t)$ such that $\mathbf{E}_{\theta} \int_{0}^{T} u_{\theta}(t)^{2} d t<+\infty$ and $\xi=\mathbf{E}_{\theta} \xi+$ $\int_{0}^{T} u_{\theta}(t) d z_{\theta}(t)$. It suffices to select $\gamma(t)=u_{\theta}(t) \widetilde{S}(t)^{-1}$. This completes the proof of Theorem 3.1

Proof of Theorem 3.2 follows immediately from the Martingale Representation Theorem applied on the probability space $\left(\Omega, \mathcal{F}_{T}^{w}, \mathbf{P}_{\theta}\right)$.

Proof of Lemma 4.1 follows from the assumptions of Theorem 3.2 and from the equation

$$
B(T)^{-1}=B(t)^{-1} \exp \left(\left[-\alpha+\rho^{2} / 2+\theta_{1}\right](T-t)-\rho\left[W_{1 \theta}(T)-W_{1 \theta}(t)\right]\right) .
$$

Proof of Remark 4.2. Let the initial wealth $c_{\theta i}=X^{(i)}(0)$ and the strategy $\left(\beta^{(i)}(\cdot), \gamma^{(i)}(\cdot)\right)$ be such that $\widetilde{X}^{(i)}(T)=\xi$ a.s. for the corresponding discounted wealth $X^{(i)}(t), i=1,2$.

Set

$$
g(t) \triangleq \gamma^{(1)}(t)-\gamma^{(2)}(t), \quad Y(t) \triangleq \widetilde{X}^{(1)}(t)-\widetilde{X}^{(2)}(t) .
$$

We have that $Y(T)=0$ a.s.. Hence

$$
Y(T)=Y(0)+\int_{0}^{T} g(t) d \widetilde{S}(t)=0 .
$$


For $K>0$, consider first exit times $T_{K}=T \wedge \inf \left\{t: \int_{0}^{t}\left(\left|\gamma^{(1)}(s)\right|+\left|\gamma^{(2)}(s)\right|^{2} d s \geq K\right\}\right.$; they are Markov times with respect to $\left(\mathcal{F}_{t}\right)_{t \geq 0}$. We have that

$$
Y\left(T_{K}\right)=Y(0)+\int_{0}^{T_{K}} g(t) d \widetilde{S}(t)=\mathbf{E}_{\theta i}\left\{Y(T) \mid \mathcal{F}_{T_{K}}\right\}=0, \quad i=1,2 .
$$

Hence

$$
0=Y(0)^{2}+\mathbf{E}_{\theta_{i}} \int_{0}^{T_{K}} g(s)^{2} \widetilde{S}(s)^{2}|V(s)|^{2} d t=0
$$

It follows that $Y(0)=0$, and $\left.g(t)\right|_{\left[0, T_{K}\right]}=0$ for any $K>0$. In addition, $T_{K} \rightarrow T$ a.s. as $T_{K} \rightarrow+\infty$. Hence $g=0$. This completes the proof of Remark 4.2,

Proof of Theorem 5.1. For any $K \in \mathbf{R}$, there exists $\theta=\theta_{K} \in \mathcal{T}$ such that

$$
\begin{aligned}
& \theta_{1} \widetilde{\sigma}+\theta_{2} \widetilde{\rho}=\widetilde{a} \\
& \theta_{1} \rho+\theta_{2} \widehat{\rho}=\widehat{V}(t)^{\top} \theta(t)=K-r+\rho^{2}+\widehat{\rho}^{2} .
\end{aligned}
$$

By Girsanov's Theorem,

$$
W_{\theta}(t)=W(t)+\int_{0}^{t} \theta(s) d s
$$

is a standard Wiener process in $\mathbf{R}^{2}$ under $\mathbf{P}_{\theta}$. We have that $d \widetilde{S}(t)=\widetilde{S}(t) V(t)^{\top} d W_{\theta}(t)$ and

$$
\begin{aligned}
d B^{-1}(t) & =B(t)^{-1}\left(\left[-r+\rho^{2}+\widehat{\rho}^{2}\right] d t-\rho d w(t)-\widehat{\rho} d \widehat{w}(t)\right) \\
& =B(t)^{-1}\left(\left[-r+\rho^{2}+\widehat{\rho}^{2}\right] d t-\widehat{V}(t)^{\top} d W(t)\right) \\
& =B(t)^{-1}\left(\left[-r+\rho^{2}+\widehat{\rho}^{2}\right] d t-\widehat{V}(t)^{\top} \theta(t) d t+\widehat{V}(t)^{\top} d W_{\theta}(t)\right) \\
& =B(t)\left(\left[-r+\rho^{2}+\widehat{\rho}^{2}\right] d t-\left(K-r+\rho^{2}+\widehat{\rho}^{2}\right) d t+\widehat{V}(t)^{\top} d W_{\theta}(t)\right) \\
& =B(t)^{-1}\left(-K d t+\widehat{V}(t)^{\top} d W_{\theta}(t)\right) .
\end{aligned}
$$

Let $\widehat{B}(t)^{-1}=e^{K t} B(t)^{-1}$. We have that

$$
d \widehat{B}^{-1}(t)=\widehat{B}(t)^{-1} \widehat{V}(t)^{\top} d W_{\theta}(t)
$$

It follows that $\widehat{B}(t)^{-1}$ is a martingale under $\mathbf{P}_{\theta}$.

Let us prove statement (i). Let $K>0$. We have that

$$
\begin{aligned}
\mathbf{E}_{\theta} \xi=\mathbf{E}_{\theta} B(T)^{-1}(\kappa-S(T))^{+} & \leq \mathbf{E}_{\theta} B(T)^{-1} \kappa=e^{-K T} \kappa \mathbf{E}_{\theta} \widehat{B}(T)^{-1} \\
& =e^{-K T} \kappa \widehat{B}(0)^{-1} \rightarrow 0 \text { as } K \rightarrow+\infty
\end{aligned}
$$


Let us prove statement (ii). Let $K<0$. We have

$$
\begin{aligned}
\mathbf{E}_{\theta} \xi & =\mathbf{E}_{\theta} B(T)^{-1}(\kappa-S(T))^{+}=\mathbf{E}_{\theta}\left(B(T)^{-1} \kappa-\widetilde{S}(T)\right)^{+} \geq \mathbf{E}_{\theta} B(T)^{-1} \kappa-E_{\theta} \widetilde{S}(T) \\
& =\kappa e^{-K T} \mathbf{E}_{\theta} \widehat{B}(T)^{-1}-\widetilde{S}(0)=\kappa e^{-K T} \widehat{B}(0)^{-1}-\widetilde{S}(0) \rightarrow+\infty \quad \text { as } \quad K \rightarrow-\infty
\end{aligned}
$$

This completes the proof of Theorem 5.1.

Proof of Theorem 5.2, Let $\theta=\theta_{K}$ and $\widehat{B}(t)$ be such as defined in the proof of Theorem 5.1

Let us prove statement (i). We have that

$$
\begin{array}{r}
\mathbf{E}_{\theta} \xi=\mathbf{E}_{\theta} B(T)^{-1}(S(T)-\kappa)^{+}=\mathbf{E}_{\theta} B(T)^{-1}(S(T)-\kappa)+\mathbf{E}_{\theta} B(T)^{-1} \mathbb{I}_{\{S(T) \leq \kappa\}}(\kappa-S(T)) \\
\leq \mathbf{E}_{\theta} B(T)^{-1} S(T)-\mathbf{E}_{\theta} \mathbb{I}_{\{S(T)<\kappa\}} B(T)^{-1} S(T) \\
\leq \mathbf{E}_{\theta} B(T)^{-1} S(T)-\mathbf{E}_{\theta} B(T)^{-1} S(T)+\mathbf{E}_{\theta} \mathbb{I}_{\{S(T)>\kappa\}} B(T)^{-1} S(T) \\
\leq \mathbf{E}_{\theta} \mathbb{I}_{\{S(T)>\kappa\}} B(T)^{-1} S(T) .
\end{array}
$$

Let $\widehat{S}(t)=e^{K t} S(t)$. By the definitions, we have that

$$
\begin{aligned}
d S(t) & =S(t)\left(a d t+V^{\top} d W(t)+\widehat{V}^{\top} d W(t)\right) \\
& =S(t)\left(a d t-V^{\top} \theta d t-\widehat{V}^{\top} \theta d t+V^{\top} d W_{\theta}(t)+\widehat{V}^{\top} d W_{\theta}(t)\right) \\
& =S(t)\left((a-\widetilde{a}) d t-\left(K-r+\rho+\widehat{\rho}^{2}\right) d t+V^{\top} d W_{\theta}(t)+\widehat{V}^{\top} d W_{\theta}(t)\right)
\end{aligned}
$$

and

$$
d \widehat{S}(t)=\widehat{S}(t)\left((a-\widetilde{a}) d t+\left(r-\rho-\widehat{\rho}^{2}\right) d t+V^{\top} d W_{\theta}(t)+\widehat{V}^{\top} d W_{\theta}(t)\right) .
$$

It follows from the standard estimates for stochastic differential equations that

$$
\sup _{K} \mathbf{E}_{\theta_{K}}|\widehat{S}(T)|<+\infty
$$

for $\theta=\theta_{K}$; see, e.g., Chapter 2 in Krylov (1980). Hence $\mathbb{I}_{\{S(T)>\kappa\}}=\mathbb{I}_{\left\{\widehat{S}(T)>\kappa e^{K T}\right\}} \rightarrow 0$ a.s. as $K \rightarrow+\infty$. By the Lebesgue Dominated Convergence Theorem, it follows that $\mathbf{E}_{\theta} \xi \rightarrow 0$ as $K \rightarrow+\infty$. Hence statement (i) follows.

Let us prove statement (ii). For $K>0$, we have that

$$
\begin{aligned}
\mathbf{E}_{\theta} \xi & =\mathbf{E}_{\theta} B(T)^{-1}(S(T)-\kappa)^{+}=\mathbf{E}_{\theta}\left(\widetilde{S}(T)-B(T)^{-1} \kappa\right)^{+} \\
& \geq \mathbf{E}_{\theta} \widetilde{S}(T)-e^{-K T} \mathbf{E}_{\theta} \widehat{B}(T)^{-1}=\widetilde{S}(0)-e^{-K T} \widehat{B}(0)^{-1} \rightarrow S(0) \quad \text { as } \quad K \rightarrow+\infty
\end{aligned}
$$


In addition, we have that

$$
\mathbf{E}_{\theta} \xi=\mathbf{E}_{\theta} B(T)^{-1}(S(T)-\kappa)^{+}=\mathbf{E}_{\theta}\left(\widetilde{S}(T)-B(T)^{-1} \kappa\right)^{+} \leq \mathbf{E}_{\theta} \widetilde{S}(T)=S(0)
$$

This completes the proof of Theorem 5.2 .

Proof of Theorem 5.3. Since the statements (i) and (ii) are equivalent, it suffices to show that (ii) holds.

Let $\operatorname{sign}(x)$ be defined such that $\operatorname{sign}(x)=1$ for $x>0$, $\operatorname{sign}(x)=0$ for $x=0$, and $\operatorname{sign}(x)=-1$ for $x<0$.

Let $\psi(t)=\left(\operatorname{sign}\left(\eta_{\theta 1}(s)\right), \operatorname{sign}\left(\eta_{\theta 2}(s)\right)\right)^{\top}$.

Let $K>0$, and let measure $Q=Q_{K}$ be selected such that

$$
W_{Q}(t)=W_{\theta}(t)-K \int_{0}^{t} \psi(s) d s
$$

is a Wiener process under $Q$. This measure exists by Girsanov's Theorem. We have that

$$
R_{\theta}=\int_{0}^{T} \eta_{\theta}(t)^{\top} d W_{\theta}(t)=K \int_{0}^{T} \eta_{\theta}(t)^{\top} \psi(t) d t+\int_{0}^{T} \eta_{\theta}(t)^{\top} d W_{Q}(t) .
$$

Let

$$
\begin{aligned}
& N_{1}(K)=\mathbf{E}_{Q}\left(\int_{0}^{T} \eta_{\theta}(t)^{\top} \psi(t) d t\right)^{2}=\mathbf{E}_{Q}\left(\int_{0}^{T}\left(\left|\eta_{\theta 1}(t)\right|+\left|\eta_{\theta 2}(t)\right|\right) d t\right)^{2}, \\
& N_{2}(K)=\mathbf{E}_{Q}\left(\int_{0}^{T} \eta_{\theta}(t)^{\top} d W_{Q}(t)\right)^{2} .
\end{aligned}
$$

We have that

$$
\inf _{K>0} N_{1}(K)=\inf _{K>0} \mathbf{E}_{Q}\left(\int_{0}^{T}\left(\left|\eta_{\theta 1}(t)\right|+\left|\eta_{\theta 2}(t)\right|\right) d t\right)^{2} \geq \underset{\omega}{\operatorname{essinf}}\left(\int_{0}^{T}\left|\eta_{\theta}(t, \omega)\right| d t\right)^{2}>0 .
$$

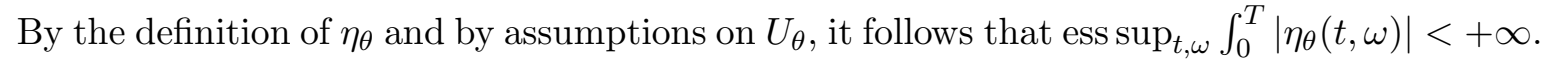
Hence

$$
\sup _{K>0} N_{2}(K)=\sup _{K>0} \mathbf{E}_{Q} \int_{0}^{T}\left|\eta_{\theta}(t)\right|^{2} d t \leq \underset{\omega}{\operatorname{ess} \sup _{\omega}} \int_{0}^{T}\left|\eta_{\theta}(t, \omega)\right|^{2} d t<+\infty .
$$

Hence $\mathbf{E}_{Q} R_{\theta}^{2} \geq K^{2} N_{1}(K)-2 K \sqrt{N_{1}(K) N_{2}(K)}+N_{2}(K) \rightarrow+\infty$ as $K \rightarrow+\infty$. This completes the proof of Theorem 5.3 .

Proof of Theorem 5.4. The statements (i) and (ii) are equivalent; it suffices to show that (ii) holds. Let $K>1$, and let $y(t)$ evolves as

$$
d y(t)=\eta_{\theta}(t)^{\top} d W_{\theta}(t), \quad y(0)=0 .
$$


Let $T_{K}=T \wedge \inf \left\{t>0: \int_{0}^{t} y(s)^{2} d s \geq K\right\}$. Let $q(t)=q_{K}(t)=\left(q_{1}(t), q_{2}(t)\right)^{\top}$ be an $\mathbb{F}$-adapted process such that $q(t)^{\top} V(t)=0, q(t)^{\top} \eta_{\theta}(t)=-K y(t)$ for $t \leq T_{K}$, and $q(t)=0$ for $t>T_{K}$. By the assumptions on $\eta_{\theta}$, it follows that there is a unique process $q$ with this properties. In addition, it follows that $|q(t)| \leq C y(t)$ and

$$
\int_{0}^{T} q(s)^{2} d s=\int_{0}^{T_{K}} q(s)^{2} d s \leq C \int_{0}^{T_{K}} y(s)^{2} d s \leq C K,
$$

where $C>0$ is defined by $\operatorname{ess~} \inf _{t, \omega}\left|\eta_{\theta}(t, \omega)\right|$.

Let a measure $Q=Q_{K}$ be selected such that

$$
W_{Q}(t)=W_{\theta}(t)-\int_{0}^{t} q(s) d s
$$

is a Wiener process under $Q$. By the definitions, it follows that

$$
d y(t)=-K y(t) d t+\eta_{\theta}(t)^{\top} d W_{Q}(t), \quad y(0)=0 .
$$

By Girsanov's Theorem again, this measure exists and is equivalent to $\mathbf{P}_{\theta}$, and, therefore, is equivalent to $\mathbf{P}$. We have that

$$
\begin{aligned}
R_{\theta} & =\int_{0}^{T} \eta_{\theta}(t)^{\top} d W_{\theta}(t)=\int_{0}^{T}\left[\eta_{\theta}(t)^{\top} q(t) d t+\eta_{\theta}(t)^{\top} d W_{Q}(t)\right] \\
& =-\int_{0}^{T_{K}} K y(t) d t+\int_{0}^{T} \eta_{\theta}(t)^{\top} d W_{Q}(t)=y\left(T_{K}\right)+\int_{T_{K}}^{T} \eta_{\theta}(t)^{\top} d W_{Q}(t) .
\end{aligned}
$$

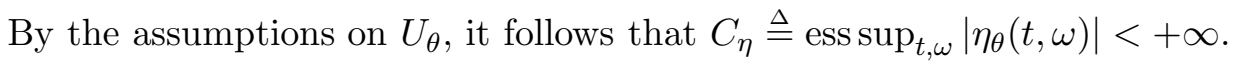

Clearly,

$$
\begin{aligned}
& \mathbf{E}_{Q} y\left(T_{K}\right)^{2}=\mathbf{E}_{Q} \int_{0}^{T_{K}} e^{-2 K\left(T_{K}-s\right)}\left|\eta_{\theta}(s)\right|^{2} d s \leq C_{\eta}^{2} \mathbf{E}_{Q} \int_{0}^{T_{K}} e^{-2 K\left(T_{K}-s\right)} d s \\
& =C_{\eta}^{2} \mathbf{E}_{Q} \frac{1-e^{-2 K T_{K}}}{2 K} \leq \frac{1-e^{-2 K T}}{2 K} \rightarrow 0 \quad \text { as } \quad K \rightarrow+\infty
\end{aligned}
$$

Consider events $A_{K}=\left\{\int_{0}^{T} y(t)^{2} d t>K\right\}=\left\{T_{K}<T\right\}$. We have

$$
\mathbf{E}_{Q}\left(\int_{T_{K}}^{T} \eta_{\theta}(t)^{\top} d W_{Q}(t)\right)^{2} \leq \mathbf{E}_{Q} \int_{T_{K}}^{T}\left|\eta_{\theta}(t)\right|^{2} d t \leq E_{Q} \mathbb{I}_{A_{K}} \int_{0}^{T}\left|\eta_{\theta}(t)\right|^{2} d t \leq T C_{\eta}^{2} Q\left(A_{K}\right) .
$$

We have that

$$
\mathbf{E}_{Q} y(t)^{2} d t \leq C_{\eta}^{2} \int_{0}^{t} e^{-2 K(t-s)} d s \leq C_{\eta}^{2} T
$$


By the Markov Inequality, it follows that

$$
Q\left(A_{K}\right) \leq \frac{1}{K} \mathbf{E}_{Q} \int_{0}^{T} y(t)^{2} d t \leq \frac{1}{K} C_{\eta}^{2} T^{2} \rightarrow 0 \quad \text { as } \quad K \rightarrow+\infty .
$$

By (A.2)-(A.3), $\mathbf{E}_{Q} R_{\theta}^{2} \rightarrow 0$ as $K \rightarrow+\infty$. This completes the proof of Theorem 5.4,

Proof of Theorem [6.1. It can be verified directly that the equations for $S$ and $B$ have the desired form.

Proof of Theorem 6.2. First, the standard Lagrange optimization techniques gives immediately that the selected $\theta$ is such that $|\theta(t, \omega)|$ is minimal over all $\theta \in \mathcal{T}$ and it is a unique solution of the problem

$$
\text { Minimize }|\theta| \text { subject to } V(t, \omega)^{\top} \theta=\widetilde{a}(t, \omega) \text {. }
$$

Further, by Martingale Representation Theorem, we have that, for some $U_{\theta} \in \mathcal{Y}_{\theta}$, presentation (3.1) holds. It was shown in Section 3 that (3.3) - (3.4) holds. By Proposition 3.1, we have that $V(t)^{\top} \eta_{\theta}(t) \equiv 0$. For our choice of $\theta$, this gives that $\theta(t)^{\top} \eta_{\theta}(t) \equiv 0$. It follows that

$$
R_{\theta}=\int_{0}^{T} \eta_{\theta}(t)^{\top} d W_{\theta}(t)=\int_{0}^{T} \eta_{\theta}(t)^{\top} d W(t)
$$

Hence

$$
\begin{aligned}
\mathbf{E} R_{\theta} \int_{0}^{T} \gamma(t) \widetilde{S}(t) V(t)^{\top} d W(t) & =\mathbf{E} \int_{0}^{T} \eta_{\theta}(t)^{\top} d W(t) \int_{0}^{T} \gamma(t) \widetilde{S}(t) V(t)^{\top} d W(t) \\
& =\mathbf{E} \int_{0}^{T} \gamma(t) \widetilde{S}(t) \eta_{\theta}(t)^{\top} V(t) d t=0 .
\end{aligned}
$$

This completes the proof of Theorem 6.2.

\section{Acknowledgment}

This work was supported by ARC grant of Australia DP120100928 to the author.

\section{References}

K. Back, Martingale pricing, Annual Review of Financial Economics 2 (2010) 235-250.

D. Becherer, The numeraire portfolio for unbounded semimartingales, Finance and Stochastics 5 (3) (2010) 327-341. 
S. Benninga, T. Bjork, and Z. Wiener, On the use of numeraires in option pricing. Journal of Derivatives 10 (2) (2002), 43-58.

F. Biagini and M. Pratelli, Local risk minimization and numeraire. Journal of Applied Probability 36(4) (1999) 1126-1139.

T.R. Bielecki, M. Jeanblanc, and M. Rutkowski, Credit Risk Modeling (Osaka University Press, Japan, 2009).

M.J. Brennan, The role of learning in dynamic portfolio decisions, European Finance Review 1 (1998), 295-306.

S.T. Cheng, On the feasibility of arbitrage-based option pricing when stochastic bond price processes are involved, Journal of Economic Theory 53(1) (1991) 185-198.

N. Dokuchaev, Two unconditionally implied parameters and volatility smiles and skews. Applied Financial Economics Letters (2006) 2 199-204.

N.G. Dokuchaev, Optimal solution of investment problems via linear parabolic equations generated by Kalman filter. SIAM J. of Control and Optimization 44 (4) (2005) 1239-1258.

N. Dokuchaev, Mathematical finance: core theory, problems, and statistical algorithms (Routledge, New York, 2007).

N. Dokuchaev, Option pricing via maximization over uncertainty and correction of volatility smile, International Journal of Theoretical and Applied Finance (IJTAF) 14 (4) (2011) $507-524$.

N. El Karoui and M. Quenez, Dynamic programming and pricing of contingent claims in an incomplete market, SIAM Journal on Control and Optimization 33 (1) (1995) 29-66.

H. Föllmer and D. Sondermann, Hedging of non-redundant contingent claims. In: W. Hildenbrand and A. Mas-Colell (eds.), Contribution to Mathematical Economics (North Holland, New York, 1986) 205-223.

H. Geman, N. El Karoui, and J.C. Rochet, Changes of numéraire, changes of probability measure and option pricing. Journal of Applied Probability 32(2) (1995), 443-458.

F. Jamshidian, Numeraire invariance and application to option pricing and hedging, working paper. http://mpra.ub.uni-muenchen.de/7167/, 2008

I. Karatzas and C. Kardaras, The numéraire portfolio in semimartingale financial models, Finance and Stochastics 11 (2007), 447-493. 
C. Kardaras, Numéraire-invariant preferences in financial modeling, Ann. Appl. Probab. 20 (2010), 1697-1728.

Lin Yee Hin and N. Dokuchaev, On the implied volatility layers under the future risk-free rate uncertainty, International Journal of Financial Markets and Derivatives (2014), in press.

Yong-Jin Kim and Naoto Kunitomo, (1999). Pricing options under stochastic interest rates: A New Approach. Asia-Pacific Financial Markets 6 (1) (1999) 49-70.

N.V. Krylov, Controlled Diffusion Processes. (Springer, New York, 1980).

M. Schweizer, A guided tour through quadratic hedging approaches, In: E. Jouini, J. Cvitanic, M. Musiela (eds.), "Option Pricing, Interest Rates and Risk Management", Cambridge University Press, 2001, 538-574.

C. G. Turvey and S. Komar, Martingale Restrictions and the Implied Market Price of Risk, Canadian Journal of Agricultural Economics 54 (2006) 379-399.

R. Weron, Market price of risk implied by Asian-style electricity options and futures, Energy Economics 30 (2008) 1098-1115. 